\title{
Tangence
}

\section{Archéologie d'une postmodernité}

\section{Frances Fortier}

Numéro 39, mars 1993

La fiction postmoderne

URI : https://id.erudit.org/iderudit/025750ar

DOI : https://doi.org/10.7202/025750ar

Aller au sommaire du numéro

Éditeur(s)

Tangence

ISSN

0226-9554 (imprimé)

1710-0305 (numérique)

Découvrir la revue

Citer cet article

Fortier, F. (1993). Archéologie d'une postmodernité. Tangence, (39), 21-36.

https://doi.org/10.7202/025750ar d'utilisation que vous pouvez consulter en ligne.

https://apropos.erudit.org/fr/usagers/politique-dutilisation/ 


\title{
Archéologie d'une postmodernité1
}

\author{
Frances Fortier
}

L'approche critique que j'adopte ici est multiple et résiste, autant que cela est possible, à une interprétation totalisante.

Karen Gould

La postmodernité a trente ans. Trois décennies de pratiques et de réflexions tentent de la définir, sans jamais dissiper le flou qui la détermine. La sédimentation discursive a presque réussi à niveler les réticences à son endroit et le mot, sinon la chose, est en train de devenir un poncif, un récit orthodoxe qui nomme le désenchantement du monde et reconnaît le métissage des cultures. La postmodernité, discrètement, devient obsolète: son pouvoir polarisateur et subversif se dissout dans l'indifférence générale et seuls quelques prosélytes tentent de donner sens à ce culte de l'inauthentique, miné par une incertitude endémique qui confine au maniérisme. Emporté par sa propre inertie, le bel éphémère aura vécu le temps de quelques colloques.

Ou l'inverse: une ultime génération postmoderne tient le haut du pavé. Qui ne pose plus son existence sur un axe temporel, mais découpe un espace d'interprétation. En font foi, entre autres, les études littéraires, qui relancent constamment la problématique $^{2}$ : synthèses, états de la question, bibliographies,

1 Ce titre n'est pas sans évoquer celui de Gérard Raulet, *Pour une archéologie de la postmodernité., Weimar ou l'explosion de la modernité, Paris, Anthropos, 1984. Comme je souscris au postulat de l'existence de régularités discursives qui gèrent les aléas du hasard, je conserve ce titre, en précisant que l'indéfini de l'article renvoie à un - fragment = pourtant bien déterminé de la postmodernité, à savoir le discours critique québécois.

2 Un regard sommaire à la production d'études québécoises sur la postmodernité dintinguerait cinq titres emblématiques - et je sais tout le périlleux d'une telle sélection - qui représentent à mon sens autant de *moments* postmodernes de ce discours critique: * Les genres et le postmodernismex, de Caroline Bayard, La mort du genre 2, actes d'un 
publications et séminaires consacrés à la postmodernité se multiplient. Canonisation ou récupération, la critique littéraire québécoise a intégré le vocable et l'utilise pour désigner une série de phénomènes hétéroclites, touchant à la fois le discours littéraire et son analyse. Et c'est précisément cet espace d'interprétation, en voie de légitimation, qui m'intéresse.

L'archéologie d'une postmodernité trouve ici son sens. Il s'agit d'examiner la postmodernité sous l'angle des régularités d'une pratique discursive, pratique qui forme un objet en reconduisant constamment une série d'énoncés recteurs ${ }^{3}$. À cet effet, cinq aspects seront interrogés - la définition de la postmodernité qui sous-tend ce discours, sa rhétorique, son lexique, l'axiologie

colloque tenu en 1987 Montréal, $N B J, \mathrm{n}^{\text {os }} 216-217,1989$, qui pose la problématique à la faveur d'une question littéraire; La camera obscura du postmodernisme de Pierre Milot, Montréal, l'Hexagone, 1988, à la visée polémique; Moments postmodernes dans le roman québécois de Janet Paterson, Ottawa, Presses de l'Université d'Ottawa, 1990, qui propose une lecture des formes postmodernes d'œuvres québécoises; Fictions de l'identitaire au Québec, de Sherry Simon, Pierre L'Hérault, Robert Schwartzwald et Alexis Nouss, Montréal, XYZ, 1991, qui suggère une lecture postmoderne de divers aspects culturels, et . Féminisme, postmodernité, esthétique de lecture: Le ...* de Karen Gould, Le roman québécois depuis 1960 (Louise Milot et Jaap Linvelt éds), Québec, Presses de l'Université Laval, 1992, qui signale l'ampleur de la visée épistémologique de la critique postmoderne. On l'aura compris, il s'agit moins ici de dresser une compilation des études postmodernes québécoises (on lira avec profit, entre autres, l'état de la question et la sélection bibliographique établie par Janet Paterson dans son ouvrage), ou de montrer les étapes d'une institutionnalisation, que de signaler l'existence de ce discours.

3 La postmodernité est un espace sans géographie et le discours québécois, il va sans dire, se nourrit d'une réflexion qui transcende les frontières: Bertens, De Man, Derrida, Fokkema, Foucault, Hassan, Hutcheon, Huyssen, Lodge, Lyotard, Rorty et Scarpetta sont les théoriciens le plus souvent convoqués et constituent un horizon commun. Pour une mise en perspective des travaux portant sur la postmodernité littéraire, on consultera l'article d'ouverture de Littérature et postmodernité (Kibédi Varga éd.), Groningue, C.R.I.N., 1986, où les - nationalités - de la postmodernité sont examinées à la lumière d'un colloque tenu en 1984 à la John Hopkins University, La traversée de l'Atlantique, colloque dont les actes sont publiés dans un numéro spécial de Critique (mai 1985). Dans ce même ouvrage, on lira aussi l'article de Leo H. Hoeck, *Indifférence, outrance et participation, dispositifs postmodernistes * qui présente - à l'occasion d'une discussion de l'ouvrage collectif sous l'égide de Fokkema et Bertens intitulé $A p$ proaching Postmodernism, Amsterdam-Philadelphie, John Benjamins Publishing Company, 1985 - un état détaillé de la question. 
qu'il révèle et son rapport au savoir-qui sont autant de propositions permettant d'assigner une visée à cet événement discursif.

\section{La postmodernité: le mot et la chose}

Ceci en guise de désignation inaugurale: le mot postmodernité, de par son intrinsèque contradiction logique ${ }^{4}$, se moule étroitement à ce qu'il entend recouvrir. Ici, l'arbitraire du signe ne joue pas et le mot est la chose. En effet, obérée de son préfixe, la postmodernité représente un défi à la cohérence sémantique, une volonté d'hétérogénéité, et un amalgame des temporalités qui sont les enjeux mêmes de la pratique postmoderne. La fécondité du terme tient précisément à cette impureté. Tout comme ce qu'il désigne, le mot annihile toute polarité et autorise le cumul d'éléments disparates.

Analogon, soit. Mais, dût-on admettre le rapprochement avec la modernité, subsiste encore la question de leur lien. En l'absence de manifeste esthétique - ce en quoi précisément la postmodernité se dégage de la logique des avant-gardes -, il demeure difficile de spécifier le rapport entre la modernité et la postmodernité. Ainsi, Antoine Compagnon, dans Les cinq paradoxes de la modernités, se fait-il le porte-parole de cette question lancinante:

4 Cette contradiction inhérente relance - et polarise - constamment la possibilité même d'une condition postmoderne. Par exemple, la position de Gianni Vattimo dans La fin de la modemité, Paris, Seuil, 1987, invite à reconnaitre dans la postmodernité un tournant authentique par rapport aux traits généraux de la modernité: «le post-moderne se caractérise non seulement comme nouveauté par rapport au moderne, mais plus radicalement comme dissolution de la catégorie de nouveau, comme expérience d'une "fin de l'histoire", et non plus comme la représentation d'un autre stade, plus progressif ou plus régressif peu importe, de cette même histoire. (p. 10). Meschonnic, dans Modernitê, modernité, Paris, Verdier, 1988, défend la position inverse: - Différence essentielle entre la modernité et le post-moderne: la modernité est une aventure de l'historicité, le post-moderne refuse d'être pensé en termes historiques. Le paradoxe est l'inversion des effets. La modernité telle que je l'entends dégagée des mythes de la rupture et du nouveau et de sa confusion avec les avant-gardes - est une trans-historicité, un indicateur de subjectivité. Sa force est de ne pas être liée à un référent fixe. D'être un mot vide, plein seulement de sujet. Le post-moderne a un référent, le moderne. Et plus il se veut ahistorique, plus il est enchaîné - (p. 226-227).

5 Antoine Compagnon, Les cinq paradoxes de la modernité, Paris, Seuil, 1990 , p. 145. 
Ihab Hassan met le mouvement de la littérature en rapport avec un phénomène social, une mutation majeure de l'humanisme occidental, qu'il n'hésite pas à appeler un changement d'épistémè ou de "formation discursive" au sens de Michel Foucault ${ }^{6}$. Ayant acquis cette légitimité philosophique, le postmodernisme s'est ensuite généralisé pour désigner tout le paysage esthétique et intellectuel contemporain, marqué par des transformations incontestables. Il n'en reste pas moins difficile de décider si le postmodernisme correspond à un authentique changement d'épistémè, ou de "paradigme" au sens de Thomas Kuhn, et s'il a donné lieu à des formes originales, ou s'il a seulement recyclé des procédés anciens dans un contexte différent. Y a-t-il continuité ou rupture avec le modernisme, et si oui, positive ou négative ? 7

Nonobstant son ancrage moderne, la question demeure pertinente et permet de spécifier l'articulation fondamentale de la postmodernité sur laquelle se fonde implicitement la critique québécoise. Il appert très nettement que ce discours récuse toute forme de polarisation et spécifie la postmodernité par son maintien d'une double posture: et moderne, mais postmoderne. J'emprunte ici fort librement à la notion de signe-transit mise de l'avant par Bernard

6 Précisons ici que ma position à cet égard se distingue de celle qui est proposée par Ihab Hassan, The Dismemberment of Oppbeus: Toward a Postmodern Literature, $2^{e}$ éd., Madison, University of Wisconsin Press, 1982 , notamment dans la mesure où il croit devoir inscrire, pour signaler un changement d'épistémè, une coupure franche entre la modernité et la postmodernité, coupure fondée sur toute une série d'oppositions telles jeu/but, anarchie/hiérarchie, présence/absence, méta phore/métonymie, paradigme/syntagme, etc. Pour une liste exhaustive, on pourra consulter l'article de Bayard, op. cit., p. 27 ou celui de Hoeck, op.cit., p. 40-41. D'autres critiques, dont Gérald Graff, Literature against Itself: Literary Ideas in Modern Society, Chicago, University of Chicago Press, 1979, préfèrent penser la postmodernité en termes de continuité.

7 La réponse à cette question pourrait être laissêe à René Payant, "Les guerriers postmodernes *, Études littéraires, vol. XIX, no 2, 1986: "C'est pourquoi je conclurai avec Jean-François Lyotard, qui a aussi assidûment fréquenté Nietzsche, en disant que dans les pratiques aujourd'hui qualifiées de postmodernes le post - " ne signifie pas un mouvement de come back, de flash back, de feed back, c'est-à-dire de répétition, mais un procès en 'ana'" - un procès d'analyse, d'anamnèse, d'anagogie, et d'anamorphose. (p. 63). Ou encore, dans une perspective opposée, à Meschonnic: "Le post-moderne est-il plus moderne que le moderne, ou anti-moderne, c'est ce que le post-moderne par lui-même ne permet pas de savoir (op. cit., p. 134). 
Andrès dans son examen de la sémiosis parodique ${ }^{8}$. Il me semble que ce qu'il fait valoir à propos des textes parodiques québécois - justement dans la mesure où la pratique postmoderne joue allègrement de la parodie - peut très bien se transposer au chevauchement des deux systèmes de modélisation en présence. S'il existe une postmodernité québécoise, elle s'appréhende souvent par l'entremise du rapport parodique qu'entretient cette littérature avec les régimes discursifs européens. Ce en quoi elle autorise une continentalisation de l'Amérique, où l'américanité se spécifie non plus à partir d'un espace mythique commun mais comme une topique. Dans cette perspective, le fait que la réflexion postmoderne soit davantage tournée vers la critique américaine prend tout son sens. C'est dans ce rapport étroit que la postmodernité, dans le discours québécois, apparaît non pas comme un avatar de la modernité, mais comme la désignation d'un espace discursif.

\section{La rhétorique du discours critique postmoderne}

En proposant une définition de la postmodernité, le présent texte souscrit au parcours discursif obligé qui caractérise la réflexion postmoderne. Tous les textes qui interrogent la postmodernité, qu'ils soient à prétention théorique ou critique, sacrifient à l'exercice. Cette entreprise de désignation passe par la discussion de l'ancrage étymologique du mot et de son rapport à la modernité. Souventes fois, on tentera de situer le moment inaugural de nomination, d'inscrire des filiations, d'établir des relais ${ }^{9}$. Il s'agit moins, semble-t-il, de réécrire l'histoire de la

8 Selon Bernard Andrès, Écrire le Québec: de la contrainte à la contrariété, Montréal, XYZ, 1990: «Le propre de cette sémiosis résiderait dans un principe de non-exclusion: non pas "ou A ou B", mais "et A et B", ou sur le mode adversatif propre à la parodie: "et $A$ mais $B$ " [...] La sémiosis parodique résiderait dans la suspension du sens entre deux systèmes placés en situation de référence adversative: et $\mathrm{A}$, mais $\mathrm{B}$ ou, comme nous le verrons plus loin: et l'Europe, mais l'Amérique. [...] D'où l'intuition pour nous, que la parodie, conçue comme opération de dérivation discursive, revêtirait aux Amériques un aspect différent (par rapport à la façon dont elle opère dans les séries littéraires européennes, plus soumises à la succession et à la hiérarchisation qu'à la juxtaposition des genres). (p. 117-118).

9 Ainsi, selon le lieu énonciatif, on attribuera la maternité du vocable à Gehlen qui a été le premier à utiliser le terme de post-histoire pour définir les traits de la modernité tardive (Vattimo, op. cit., p. 108, et 
postmodernité à chaque prise de parole que de mettre en évidence l'indétermination du sens.

Au delà des divergences, un argument consensuel affleure: l'existence d'une pratique artistique postmoderne n'est jamais mise en doute. Peu importe le flou qui perdure autour de la notion de postmodernité, on distingue la spécificité d'une telle pratique. Dans le champ littéraire, l'attribution ${ }^{10}$ s'appuie sur un inventaire de paramètres qui autorisent la lecture et surtout la relecture. Souvent informée par une approche textuelle, la critique qualifie de postmoderne tout texte qui, dans le cadre d'une fiction "réaliste", démultiplie la voix narrative, inscrit la figure du locuteur et joue d'abondance des structures autoreprésentatives, qu'il s'agisse de la mise en abyme, de la surformalisation, de la parodie ou de l'intertextualité. Ces critères, auxquels s'ajoutent l'indétermination spatiotemporelle et l'intégration de genres dits mineurs, découpent un espace textuel où ce qui importe est moins la présence d'un procédé isolé que le cumul de tous les éléments du paradigme. La facture postmoderne

Meschonnic, op. cit., p. 220); ou à Francis Ponge, en 1954 (KremerMarietti, "La postmodernité qui vient à nous*, Littérature moderne 1, Paris-Genève, Champion-Slatkine, 1988, p. 185); le plus souvent, on recourra à Lyotard, dont La condition postmoderne: rapport sur le savoir, Paris, Minuit, 1979, a été commandé par le gouvernement du Québec [p.9]. Le moment fondateur et incontournable semble être le débat Harbermas-Lyotard, sans cesse reconduit (Milot, op. cit., Rorty, -Habermas, Lyotard et la postmodernité, Critique, $n^{\circ} 442$, 1984). Cette volonté de nommer la postmodernité autorise des reconversions: ainsi, Scarpetta, après avoir contribué à la fortune du mot dans L'impureté, Paris, Grasset, 1985, le discrédite dans un ouvrage ultérieur, L'artifice, Paris, Grasset, 1988, et propose d'y substituer le baroque. On pourrait aussi faire valoir, comme un des moments qui relancent l'intérêt québécois à l'endroit de la postmodernité, le colloque de 1982, conservé sur bande vidéo, "La notion d'impureté et le postmodernisme*, qui donne lieu à une discussion entre Scarpetta et Payant.

10 Je travaille ici avec les termes de Foucault lorsqu'il propose - dans Les mots et les choses, Paris, Seuil, 1966 - , le quadrilatère du langage pour analyser le travail de représentation à l'âge classique: désignation, attribution, articulation et dérivation. L'attribution est redevable du verbe être: «Il y a proposition - et discours - lorsqu'on affirme entre deux choses un lien d'attribution, lorsqu'on dit ceci est cela* (p. 158). Par analogie, le discours critique dit *ceci est postmoderne ». D'autre part, le jeu de la dérivation a fait que les appellations "postmodernisme *, "postmoderne * ou "postmodernité * se sont stabilisées, du moins dans les études littéraires, au profit des vocables "postmodernité " et "postmoderne, sans trait d'union. 
réside dans l'exploitation d'une structure d'indécision qui sature tous les plans du texte, indécision qui tient à la perversion systématique du statut des actants de la narration, à la dé-réalisation subtile de l'espace diégétique et au questionnement explicite de l'enjeu de la fiction.

D'où la possibilité d'intégrer au corpus postmoderne, en dehors d'une appréhension strictement temporelle, des ouvres qui avaient été lues autrement auparavant: à l'analyse d'œuvres strictement contemporaines s'ajoute de fait la recatégorisation de textes dont on reconnaît l'effet postmoderne ${ }^{11}$. La critique postmoderne, qu'on appelle parfois paracritique ou métacritique, opère un découpage différent de la réalité littéraire, traversant les époques et les genres littéraires, les littératures nationales et les clivages lecture/écriture, négligeant la distance entre la création et la critique, pour créer un espace discursif de dispersion. Le vecteur premier de regroupement sera néanmoins celui d'une sensibilité particulière, que Karen Gould reconnait chez Brossard mais qui traduit tout autant l'attitude critique postmoderne:

Sensibilité postmoderne qui semble s'exprimer par certains procédés narratifs, par une notion de l'écriture comme lecture et déplacement du sens, par sa déligitimation du métarécit de la science et par son discours sur l'histoire. (p. 195)

11 Je pense ici notamment aux travaux de Linda Hutcheon, A Poetics of Postmodernism: History, Theory, Fiction, New York et Londres, Routledge, 1988, et à ceux de Janet Paterson, op. cit., qui, d'entrée de jeu, proposent des sélections relativement hétéroclites, allant de Beckett à Rushdie, en passant par Barth, Barthelme, Marquez, Eco, Butor, Calvino, Borgès, Ondaatje, Sarraute et Nabokov, auxquels il faudrait sans doute ajouter Auster. Pour sa part, la fiction postmoderne québécoise est représentée par Aquin, Bessette, Brossard, Ducharme, Villemaire, Godbout, Ouellette-Michalska et Poulin. L'ouvrage de Fokkema et Bertens, op. cit., ajoute au corpus: Pynchon, Coover, Sontag, Vonnegut, Hawkes, Sukenick, Brautigan, Cortazar, Fuentes, Fowles, Murdoch, Lodge, Handke, Strauss, Bernhard, Rosei, Aksënov, Amalric, Vampilov, Krol, Michiels et de Winter. Pour une réaction à l'étiquette postmoderne, on lira le texte de John Barth, "La littérature du renouvellement", Poétique, $\mathrm{n}^{\circ} 48$, novembre 1981, p. 397, où il précise que ses propres romans lui semblent posséder à la fois les caractéristiques du modernisme et celles du postmodernisme, tout en proposant sa propre liste d'auteurs postmodernes et celui d'Eco, Apostille au "Nom de la rose, Paris, Librairie générale française, 1987, qui distingue chez Joyce des ouvrages modernes et des ouvrages postmodernes (p. 68). 
La sensibilité postmoderne dont il est question ici est commune à la réflexion et à la pratique postmodernes. La participation du lecteur au jeu de réécriture et l'activation du volet spéculaire de toute écriture, fondamentales dans la pratique postmoderne, essaiment du côté du discours critique dans un double jeu de reconnaissance mutuelle. Car s'il est une particularité de ce discours critique, c'est bien dans la postulation implicite d'un ethos postmoderne qu'il faut la situer ${ }^{12}$. C'est en ce sens que Linda Hutcheon parle d'une poétique de la postmodernité, qui englobe à la fois le discours critique et la fiction, en une même sensibilité complice.

Une autre voie tout aussi largement consensuelle qui émerge du discours critique est l'articulation socioculturelle de cette littérature: de fait, la postmodernité ne se pense pas en dehors du social. Ainsi, le retour du lisible, selon Régine Robin, rend caduque la logique de distinction bourdieusienne ${ }^{13}$. Ce renversement n'est pas le seul suscité par la postmodernité; la mise en rapport constante avec la "réalité " ambiante - et Robin dira que la postmodernité "cherche à théoriser la réalité des années 1980 " (ibid., p. 10) - dénote une approche globalisante qui menace, à mon sens, la spécificité du littéraire.

D'autant plus que ce rapport à la réalité, déficitaire semblet-il, fonde la pensée postmoderne. En effet, selon Vattimo, ce sont avant tout les qualifications de sujet et d'objet qui ont constitué le cadre dans lequel s'est consolidée la notion même de réalité:

12 Cet éthos découle, semble-t-il, de la reconnaissance, mieux, de la construction, par le lecteur d'une structure textuelle adéquate. On aura reconnu ici la définition de l'ethos poétique du Groupe $\mu$, qui pourrait servir de modèle analogique.

13 Régine Robin, dans . Le retour du lisible dans la littérature française aujourd'hui ", Cabiers de rechercbe sociologique, $\mathrm{n}^{\circ} 12$, printemps 1989 , dira que la distribution de la légitimité qui allait du haut vers le bas est * un état de choses aujourd'hui totalement dépassé. (p. 64). Robin précise encore, cette fois dans la "Présentation de ce numéro intitulé "L'énigme du texte littéraire que *le postmodernisme veut opérer un retour vers la lisibilité, vers le public, et ne craint pas de s'insérer dans les structures du marché. [...] Néanmoins, dans ce retour du lisible, le postmodernisme réinscrit une part des anciens jeux de langage de l'avant-garde, en parodiant, pastichant les anciennes formes littéraires.. Cette affirmation sert de préambule à une mise en perspective des diverses conceptions de la postmodernité, notamment celles d'ihab Hassan, Fredric Jameson, de David Lodge, de Susan Suleiman et de Linda Hutcheon [p. 8-9]. 
Par la perte de ces déterminations, l'homme comme l'être entrent dans un cadre oscillant [schwingendl, que l'on doit imaginer à mon avis comme le monde d'une réalité *allégée * car moins nettement divisée entre le vrai et la fiction, l'information ou l'image: monde de la médiatisation totale où nous nous situons déjà pour une bonne part. (op. cit., p. 185)

Comme si la question de la postmodernité imposait la nécessité de penser globalement le réel. Ce travail de reconnaissance d'une réalité postmoderne, mené de front par la critique littéraire, la sociologie et la philosophie, demeure toutefois constamment nuancé par une attitude interrogative. Marquée par un déni de la théorisation, qu'on aurait tort de confondre avec une pétition d'anti-intellectualisme, la postmodernité critique prétend s'exercer à partir d'un lieu de connivence. Dans cette perspective, la critique déconstructionniste représente l'illustration parfaite de l'attitude postmoderne. Walter Moser, dans sa typologie en trois volets des discours critiques - romantique, moderne et déconstructive retient la stratégie de complicité comme critère déterminant de cette "critique du troisième type":

Le travail de la critique déconstructive ne poursuit pas la mise à distance ou - ce qui est son équivalent inversé - l'appropriation triomphante de son objet sur le mode agonique. II accepte au contraire de s'engager avec son objet, ne craignant pas le contact direct avec lui, n'évitant pas le corps à corps avec le discours critiqué. Pourtant, ce corps à corps est moins de la lutte que de la connivence. Le critique a cessé d'aspirer, en matières discursives, à une séparation nette entre sa pratique et son objet, que cette séparation soit pensée selon la modalité de la hauteur ou de l'extériorité. Il se sait toujours déjà engagé dans une relation de complicité avec son objet et accepte cette relation d'entrée de jeu ${ }^{14}$. (op. cit., p. 135)

S'il existe bien une stratégie rhétorique de la critique postmoderne, elle passe nécessairement par les trois lieux argumentatifs

14 Walter Moser, toujours dans * Réinscrire, déconstruire: une critique du troisième type *, $R S / S I$, vol. X, nos $1-2-3,1990$, spécifie cette relation en la rapprochant de la pratique parodique littéraire: "La déconstruction, et avec elle l'enjeu d'une critique postmoderne, a partie liée avec la parodie, dans la mesure où on est prêt à soustraire cette dernière à une conceptualisation exclusivement moderne. La critique déconstructive a ainsi adopté comme une de ses caractéristiques ce qui a toujours été à la base même de l'écriture parodique, quel que soit son investissement agonistique: la position critique est élaborée avec et dans les matériaux de l'autre texte." 
que nous venons d'explorer: un consensus sur l'existence d'une pratique postmoderne sur la foi de critères formels, un rapport à la réalité qui tend à une appréhension globale du phénomène et, enfin, une position énonciative complice qui participe activement à la création d'une espace d'interprétation. C'est en ce sens que la critique postmoderne apparaît comme une rbétorique de l'adbésion, portée par une attitude de lecture qui se définit elle-même en termes d'esthétique.

\section{Le lexique de la postmodernité}

Pensée en termes de pratique de lecture, la critique postmoderne n'est pas dupe, pour autant, de son adhésion à l'objet: rusée, consciente de ses mécanismes, la critique phagocyte délibérément les enjeux de l'écriture postmoderne. Le lexique, à cet égard, est fort éloquent: un inventaire fermé de termes, relativement sédimentés, construisent une définition implicite de la postmodernité comme une investigation sceptique de la diversité du monde. Les trois paradigmes esquissés ci-après - hétérogénéité, incrédulité et regard - sont autant de bases classématiques qui médiatisent le rapport entre la théorie et la fiction postmodernes.

Ainsi l'bétérogène, sous sa forme substantivée, devient le point focalisateur d'une praxis qui reconnaît et reconduit à la fois le métissage culturel des sociétés contemporaines, le mélange des formes textuelles canoniques et marginales, l'impureté des styles et des régimes discursifs, la contamination générique - dans sa version identitaire ou littéraire-, la fragmentation du sujet et le discontinu historique et diégétique.

De fait, porté par la conviction que le sens de l'histoire est redevable uniquement des systèmes d'intelligibilité qui l'interprètent, le discours postmoderne est foncièrement incrëdule: devant les métarécits et les visions totalisantes, il doute, interroge, toujours sceptique à l'endroit des autorités légitimantes. Cette mise à distance teintée d'ironie s'exerce, dans la même mesure, à ses propres dépens: ce discours cumule les précautions énonciatives et les modalisations - peut-être, si, autant que cela est possible, semble-t-il - en un refus systématique de la synthèse, de la clôture et de la notion de vérité ${ }^{15}$.

15 Par exemple, depuis le début de cet article, je repousse à la marge la tentation du paradoxe, dans la mesure où je prétends donner à lire la 
Cette réticence est condition de son regard. Ce regard de la civilisation de l'image et du spectacle est sensible à l'illusion, à l'artifice, à la réduplication, à la dé-réalisation, au simulacre, à la spécularité. C'est dans l'interstice entre le miroir et le réel que la postmodernité enclenche ses mécanismes autoreprésentatifs en une mise en abyme à l'infini qui reflète à la fois l'écriture narcissique qu'elle examine et l'esthétique de la lecture qu'elle propose.

\section{Esquisse d'une axiologie}

Un tel vocabulaire est-il l'exact reflet des clichés apocalyptiques qui définissent nos cultures anomiques en termes d'amnésie collective, d'éclatement des structures sociales, de déperdition généralisée du sens? Ou n'est-il pas plutôt la manifestation d'un procès axiologique ${ }^{16}$ en voie d'élaboration? Quelles seraient dès lors les valorisations qui s'érigent en préférables?

À première vue, la postmodernité littéraire s'apparente à une esthétique de la négation. Négation dans la mesure où elle récuse le novum qui est la clé de voûte de la modernité. Ainsi, la parodie et son cortège de manipulations intertextuelles viennent déboulonner le double principe à la base de la valorisation littéraire, à savoir celui d'un sujet propriétaire exclusif de son discours et qui se doit d'engendrer une ouvre absolument unique et originale, en rupture totale avec l'esthétique qui l'a précédée. A contrario, la postmodernité valoriserait le recours

doxa postmoderne. La valeur donnée, jamais discutée est celle d'une condition postmoderne, d'une ère postmoderne, d'une sensibilité postmoderne. L'entreprise est donc vouée à la reconduction qui en est la condition essentielle. Cette affirmation n'est pas une évaluation, mais un constat, et la position énonciative que j'adopte est celle de la tautologie, puisque c'est le seul bors-lieu possible de mon propre discours.

16 La lecture axiologique cherche dans le discours des indices d'un système pré-construit qui informe les mécanismes de valorisation. Dans un article récent, Joseph Melançon, "Le travail de l'axiologie dans Agonie de Jacques Brault., Le roman québécois depuis 1960, op. cit., ramène l'axiologie vers l'énoncé, ce qui lui permet de préciser qu'il ne s'agit pas de chercher le non-dit ni même le présupposé: -Je pense toujours que l'axiologie est un paradigme pré-construit de valeurs réifiées qui se décèlent dans le discours par des inférences sémantiques, figuratives et argumentatives. J'aimerais ajouter, tout de même, que cette axiologie m'apparaît, aujourd'hui, relever de l'énoncé, non du signifié. (p. 79). Il va sans dire que j'endosse ici cette position. 
ludique et distancié aux formes déclassées et serait porté par un épigonisme qui "l'empêche avant tout de posséder un style propre, au point de le contraindre à puiser les formes de son art, de son architecture et de la mode, dans ce grand dépôt de costumes théâtraux qu'est devenu pour lui le passé " (Vattimo, op. cit., p. 170).

La valeur positive d'un tel épigonisme fonde la postmodernité: la mémoire postmoderne n'est jamais judicative et privilégie la juxtaposition plutôt que la dialectique. Pareillement, la sollicitation du présent est tributaire d'une volonté de niveler le clivage entre les codes majeurs et les modes mineurs de la culture ambiante. Les dichotomies usuelles qui fondent la modernité littéraire - réalité / fiction, sujet/objet, lisible/scriptible, lecture/écriture, théorie/fiction, vrai/faux, sérieux/ludique, illusion/réalité - sont annihilées au profit d'une dynamique de l'amalgame qui les réinscrit, autrement et tous ensemble, dans une parole dont l'intentionnalité ultime permet une lecture du sens $^{17}$.

La vision postmoderne, aussi fragmentée soit-elle, balaie en larges faisceaux son héritage culturel et signale toujours sa position de sujet regardant lorsqu'elle redécoupe le champ littéraire, se réapproprie des formes textuelles et réinvestit des registres autrement négligés. L'incrédulité fonde la différence: là où la modernité obéit à une stratégie de la tabula rasa et postule une téléologie dont elle serait l'achèvement, la postmodernité et ses métafictions proposent un système d'intelligibilité de l'histoire où la conscience du simulacre permet de reconnaître les processus de construction du sens qui légitiment la lecture du passé. En ce sens, la postmodernité relève d'une esthétique de la récupération et de l'intégration qui sanctionne un déplacement des valeurs de la modernité, tout entière fondée sur la légitimité de la distinction et de l'exclusion.

C'est précisément là où une telle axiologie se polarise: d'une part on déplore ce baroquisme qui aplatit la culture et on le

17 Scarpetta définit en ces termes l'enjeu de la postmodernité: "Le mot d'ordre moderniste était le refus de l'illusion. Le postmoderne est une revendication des procédés de l'illusion, non pour produire un leurre, mais pour faire triompher les simulacres. Pousser l'illusion à son paroxysme, jusqu'au point où c'est la réalité même qui apparaît comme une illusion - (op. cit., p. 63). 
dénonce comme une idéologie de la société de consommation; d'autre part, on admet l'impossibilité d'être en dehors de l'idéologie et on prétend lutter de l'intérieur contre l'idéologie dominante. En un face à face irréductible, on oppose le kitsch à la conscience critique. Le discours postmoderne est porté par cette opposition fondamentale qui autorise le partage clanique du champ ${ }^{18}$ : peu importe qu'on endosse la postmodernité ou qu'on la récuse, la reconnaissance d'un "état de fait " postmoderne représente l'articulation enthymématique de tous ces textes. S'il fallait en outre signaler le paradoxe de la postmodernité - et il semble qu'il faille absolument sacrifier à la mode qui gère l'appréhension du phénomène - , je le situerais exactement dans cette stratégie de récupération qui dénonce les systèmes totalisants en rapatriant, en une critique qui se sait totalisante, le spectre entier des phénomènes postmodernes, qu'ils soient d'ordre esthétique, ontologique ou épistémique. De fait, la postmodernité est le lieu d'intégration de discours divers, agoniques ou confortants, qui postule une interdépendance étroite entre tous les aspects du réel, qu'ils relèvent de l'identité, de la culture, de la politique, du savoir, et qui rapatrie toutes les formes de marginalisation sociale ou littéraire en une assomption de l'Autre, de la différence, du passé, des aspects mineurs du présent, de la réflexion théorique. La postmodernité est une écologie ${ }^{19}$.

\section{Modèle heuristique ou fiction régulatrice?}

À la fois l'un et l'autre, et consciente de l'être. La tendance à concevoir la postmodernité comme une décadence où domine la pensée du frivole ne résiste pas à l'examen. Une pensée sans concept n'est une "pensée faible" que dans une intentionnelle

18 Comme en fait foi le débat Baudrillard/Hutcheon (Hutcheon, op.cit., p. 222-225). Si on voulait délimiter une frontière qui se joue des obédiences dogmatiques - et je sais ce que ces associations ont de far felu - , on distinguerait une filiation qui va de Baudrillard à Compagnon en passant par Jameson et Meschonnic et une autre qui rallie Hutcheon, Said, Rorty et Vattimo.

19 Est-il si facile d'éviter les pièges de la doxa? La tentation de la paralogie est inévitable et on ne peut que buter ici sur l'alignement idéologique d'une telle éthique, qui préconise les valeurs consensuelles de la cité démocratique concernant la conscience critique, l'hétérogénéité, la participation au réel, etc. 
mise à distance des systèmes de légitimation. La postmodernité est un point de vue qui mobilise toutes les disciplines des sciences humaines et questionne à la fois l'individu, le social, l'ontologique, la pratique esthétique. Dans le champ littéraire, son rapport au savoir contemporain passe par trois points majeurs, à savoir le poststructuralisme, la pensée féministe et la déconstruction. Il ne s'agit pas ici d'identifier l'épistémè qui autoriserait une telle convergence mais de tracer des corrélations archéologiques ${ }^{20}$.

La question du sujet est sans doute un des axes de problématisation majeur de la réflexion contemporaine: ce sujet, débouté par la pensée structurale et la psychanalyse, n'existe pas hors du langage, et encore n'est-il qu'une position énonciative, virtuellement multiple. Le poststructuralisme, en acquiesçant à ce décentrement du sujet, refuse la clôture de l'énoncé et la résolution des sens contradictoires, admet l'indétermination de ce sujet et de son rapport au sens. La déconstruction, comme programme de lecture qui postule un décalage entre l'énonciation et l'énoncé où s'incrit le doute, affirme que le sens ne peut résider dans un projet énonciatif global et assignable, et se veut une critique à la fois de la représentation et de son contenu de vérité. La réflexion féministe, tentée un temps par une adéquation du sujet littéraire et du sujet social qui viendrait sanctionner l'accession de la femme au statut de sujet, achoppe nécessairement sur cette butée mais redonne son pouvoir de contestation au discours par sa subversion de la légitimité du métarécit patriarcal ${ }^{21}$.

20 Aux fins de la présente esquisse, je ne retiens que certains axes de problématisation communs. Il va sans dire que chacune de ces pratiques discursives, en soi foyer de débats, nécessiterait une lecture archéologique approfondie. Seule une distance temporelle suffisante autoriserait l'affirmation d'une épistémè postmoderne qui gèrerait l'ensemble de ces pratiques discursives.

21 Les liens du féminisme et de la postmodernité ne sont cependant pas si univoques et font l'objet d'une pléthore de réflexions théoriques. À ce sujet, on lira Barbara Havercroft, (Protée, vol. XX, n 3, 1992, p. 52). D'autre part, en contexte québécois, la tentative de Louise Dupré (Stratégie du vertige, Montréal, Éditions du Remue-ménage, 1989) d'inscrire la postmodernité dans la foulée du féminisme se voit modalisée à la fois par Bersianik et Brossard qui distinguent nettement la postmodernité mâle: toutes deux récusent une postmodernité nihiliste, négative, et l'accusent d'être un terme phallocrate qui ne peut servir les prétentions positives féministes. Le même regard sera posé sur le Quand je lis je m'invente de Suzanne Lamy, Montréal, l'Hexagone, 
Relayées dans le discours postmoderne, ces problématiques se traduisent par l'autoreprésentation d'un sujet incrédule qui refuse de penser la différence en termes d'opposition et préfère la juxtaposition. En effet, la spécificité de la postmodernité est son pluralisme: loin d'opérer la synthèse, elle fait floculer les savoirs. Au delà des divergences, la critique littéraire québécoise postmoderne cherchera dans les textes des positions énonciatives distinctes, montrera leur pluralité et leur incohérence, et dépistera l'intertextualité. Elle propose des relectures, suggère l'ouverture du corpus "national" aux paroles migrantes, et redécouvre la parole du sujet ${ }^{22}$. La critique littéraire a (presque) toujours été concernée par les médiations sociale et historique des ouvres; la différence ici, c'est qu'il s'agit de penser le présent non pas dans les termes du passé, mais dans la réalité présente, qui a tout du simulacre.

Dans sa spécificité énonciative cette fois, on pourrait faire valoir, avec Meschonnic, que le discours postmoderne n'est pas un discours mais un acte:

Le discours du post-moderne mime le post-moderne. Il est jeu de langage, et il est pragmatique, non seulement en ce qu'il se veut homogène à ce qu'il décrit, mais en ce qu'il est un performatif du post-moderne. Il fait ce qu'il dit dans l'acte de le dire. Son énonciation l'accomplit. Il en est la raison, et la rhétorique. (op. cit., p. 246)

Cependant, cette dimension pragmatique du discours postmoderne, même si on l'admet aisément, ne m'apparaît pas un critère suffisamment discriminant pour le spécifier. Que la postmodernité soit un lieu d'énonciation emblématique, qui sert davantage à

1984, - qu'on pourrait aisément reconnaître comme le premier discours critique postmoderne québécois dans la mesure où il propose une position énonciative en prise directe sur la postmodernité - et on se sentira obligé de fonder la distinction: -D'après Françoise Collin, c'est quand il se fait revendicateur que le féminisme se définit comme moderne; c'est lorsque, court-circuitant la biérarchie et l'opposition binaire des sexes, il installe partout la différence et l'bétérogénéité qu'il répond à l'appel du post-moderne. (Sherry Simon, .Suzanne Lamy: le féminin au risque de la critique *, Voix et images, $n^{\circ} 37$, automne 1987.)

22 Ainsi, on ne s'étonnera pas du déplacement de l'intérêt: un temps fascinée par les dialogismes intertextuels, la critique se tourne présentement vers la littérature intime, qui focalise à elle seule l'ensemble de la réflexion postmoderne en termes de sujet, de récit, de faux, de simulacre, d'indécision, de stratégies de lecture. 
conforter une posture critique qu'à engendrer une praxis autre, n'a rien pour surprendre: à ce titre, l'ensemble des discours critiques se range sous une telle définition. La "réalité " de la postmodernité n'existe pas plus que celle de la modernité: les objets de savoir sont des faits de discours, et l'affirmation de Meschonnic relève, elle aussi mais sans le dire, d'une pratique régulée.

Je préfère concevoir la postmodernité comme un champ discursif spécifié, en train de s'élaborer, élaboration qui passe nécessairement par la reconduction d'une série minimale d'énoncés recteurs. Leur cumul atteint le seuil de positivité et la postmodernité acquiert les caractéristiques d'une pratique autonome régie par un faisceau de régularités. La postmodernité est un regard qui distingue des objets, une rbëtorique qui prescrit une position définie à tout sujet, un discours qui crée un champ d'utilisation et de stabilisation de concepts, une stratégie performative consciente de ses mécanismes, et une spéculation heuristique qui propose une saisie du réel.

\section{Zerbrechen: bref dialogue devant un miroir}

Vous nous avez ramenés au point de départ. Soit. Pourquoi, dès lors, nous avoir fait parcourir tant d'espaces disparates? Nous vous suivions, dans l'espoir de tout savoir sur la postmodernité: au terme de votre discours, nous demeurons ignorants, et incrédules. Croyez-vous avoir réussi à fonder votre discours, et à prouver l'existence de la postmodernité?

À ces questions, je crains bien que celui que j'imite aurait répondu qu'il n'y a pas de réponse possible. J'ai tendu un miroir devant un espace discursif, et la réflexion a produit une série de signes qui ne sont peut-être que des simulacres. Mais c'était pour mieux vous faire voir, mon enfant. Au fait, qui a dit que voir était savoir? 